\title{
Avances y retos de la bioética en Honduras.
}

\section{Progress and challenges of bioethics in Honduras.}

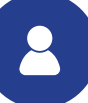

\section{Eleonora Espinoza 1}

https://orcid.org/oooo-0oo1-7218-3481

${ }^{1}$ Universidad Nacional Autónoma de Honduras, Facultad de Ciencias Médicas, Unidad de Investigación Cientifica, Comité de Ética en Investigación Biomédica, Tegucigalpa, Honduras.

\section{PALABRAS CLAVE}

Ética, Ética Institucional, Comités de Ética, Ética en la Investigación Científica.

\section{KEYWORDS}

Ethics, Ethics Institutional, Ethics Committees, Scientific Publication Ethics.

\section{CITAR COMO}

Espinoza E. Avances y retos de la bioética en Honduras. Rev. cienc. forenses Honduras. 2021; 7(2): 75-80. doi:10.5377/ rcfh.v7i2.13197

\section{AUTOR PARA CORRESPONDENCIA}

Dra. Eleonora Espinoza:

eleonora.espinoza@unah.edu.hn

\section{HISTORIA DEL ARTÍCULO}

Recepción: 1 -11- 2021

Aprobación: 17 -11- 2021

\section{DOI}

https://doi.org/10.5377/ rcfh.v7i2.13197

DECLARACIÓN DE RELACIONES $Y$ ACTIVIDADES FINANCIERAS $Y$ CONFLICTOS DE INTERÉS

Ninguna

\section{RESUMEN}

Honduras no cuenta con una regulación nacional sobre la ética de la investigación, que le permita establecer una comunicación más fluida entre los distintos actores ligados a la investigación nacional, estandarizar los procedimientos de revisión y velar de manera más adecuada por la seguridad de los participantes. Es imprescindible que las autoridades de la Secretaría de Salud como ente rector de la salud en el país, comprendan la importancia de la ética y la bioética, gestión que hasta ahora ha asumido la academia través de las universidades públicas y privadas. Como ejemplo destacado citamos el trabajo realizado por el Comité de Ética de Investigación Bioética de la Facultad de Ciencias Médicas de la Universidad Nacional Autónoma de Honduras, que impulsó en los últimos años la creación de nuevos comités y fortaleció los ya existentes, impulso la conformación de la Red de Comités de Ética en Investigación de Honduras, y la participación en una Red de Comités de Ética Regional de Centro América y el Caribe y recientemente la creación de la Comisión Nacional de Bioética. Es indudable la importancia de trabajar en 


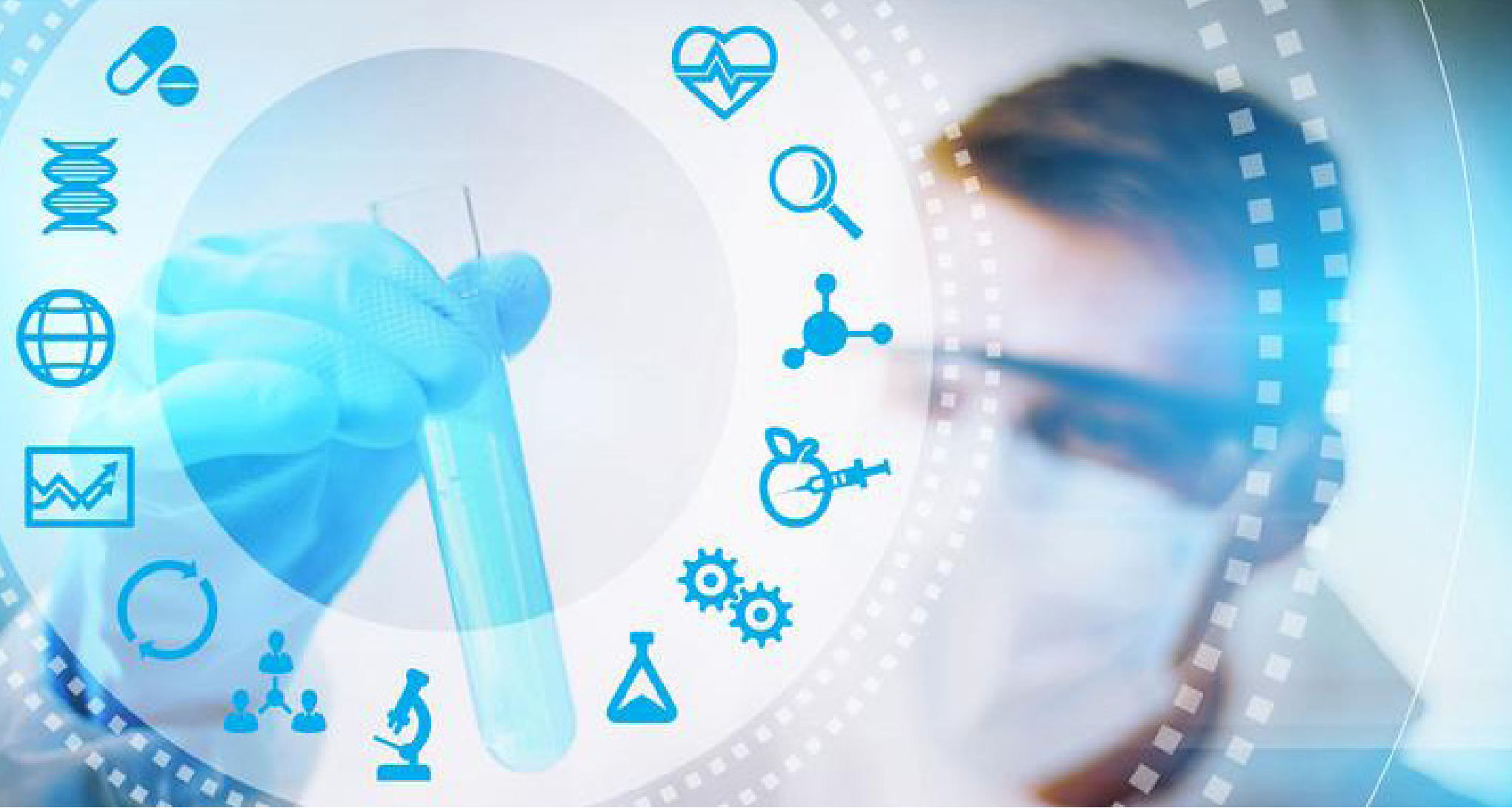

redes nacionales como internacionales en situaciones de salud de emergencia como la que vivimos actualmente con la pandemia COVID-19, en este marco se han dado un sin número de dilemas éticos que obligaron a los comités de ética a adoptar cambios en sus procesos de revisión siendo más flexibles; acortando los tiempos de revisión, aumentando las jornadas laborales de sus miembros, así como la implementación de herramientas de revisión en línea. A fin de consolidar el crecimiento de la bioética como pilar fundamental de las ciencias e investigación es necesario tener una legislación que permita la gobernanza de la ética y bioética de la investigación en el país, siendo el fin prioritario garantizar la protección de los derechos de las personas sujetas de investigación.

\section{ABSTRACT}

Honduras does nothave a national regulation on research ethics, which would allow it to establish a more fluid communication between the different actors involved in national research, standardize review procedures, and ensure the safety of the participants in a more adequate manner. It is essential that the authorities of the Ministry of Health, as the governing body of health in the country, understand the importance of ethics and bioethics, a task that until now has been assumed by the academia through public and private universities. As an outstanding example, we cite the work done by the Bioethics Research Ethics Committee of the Faculty of Medical Sciences, which in recent years has promoted the creation of new committees and strengthened existing ones, the formation of the network of research ethics committees in Honduras, participation in a network of regional ethics committees in Central America and the Caribbean and most recently the creation of the National Bioethics Commission.It is undoubtedly important to work in national and international networks during emergency health situations such as the one we are currently experiencing with the COVID-19 pandemic. In this context, there have been several ethical dilemmas that have forced ethics committees to adopt changes in their review processes by being more flexible, such as shortening review times, increasing working hours of its members, as well as the implementation of 
online review tools. In order to consolidate the growth of bioethics as a fundamental pillar of science and research, it is necessary to have legislation that allows the governance of ethics and bioethics of research in the country, with the priority aim of guaranteeing the protection of the rights of research subjects.

\section{INTRODUCCIÓN}

Honduras, a la fecha no cuenta con una regulación en ética de la investigación, las actividades de investigación responden a la visión institucional o de los investigadores y en la mayoría de los casos no se abordan problemas de importancia nacional. El 4 noviembre de 2020, apareció publicado en la Gaceta No.35,419 (medio escrito de comunicación oficial del Estado de Honduras) el acuerdo No. 0412020 sobre ensayos clínicos (https://arsa.gob.hn/ public/archivos/AcuerdoNo.041-2020-Ensayos...) "Disposiciones para la Regulación y Control Sanitario de los Ensayos Clínicos en Seres Humanos o Muestras Biológicas de éstos en Fase I, II, III y IV y las cuotas de recuperación por servicios prestados", Artículo 1.Del Registro de Comité Ético Científico (RCEC), en la Agencia de Regulación Sanitaria (ARSA) ${ }^{1}$. Ley creada más con fines mercantiles que regulatorios, que deja a discreción de cada comité tener su propio manual de procedimientos lo que no permite estandarizar la revisión, ni qué tipos de estudios deben ser sometidos a dictamen ético en contraposición a lo recomendado en la Declaración de Helsinki y en el Consejo de Organizaciones Internacionales de las Ciencias Médicas (CIOMS), que establecen que todo protocolo de investigación debe ser sometido para dictamen ante un comité de ética ${ }^{2,3}$. Es pues necesario en nuestro país cambiar esa visión fragmentada y personalizada por una visión integral, inclusiva de todos actores involucrados, para lograr la gobernanza en el tema de la ética y de la bioética de la investigación.

\section{Experiencia en Honduras}

Desde la Facultad de Ciencias Médicas (FCM) de la Universidad Nacional Autónoma de Honduras (UNAH), se ha impulsado la creación de comités de ética institucionales, función que debería rectorar la Secretaria de Salud, es así como en el 2000 comienza a funcionar el Comité de Ética en Investigación Biomédica (CEIB) de la Facultad de Ciencias Médicas el cual cuenta con registro vigente de inscripción de la Oficina Federal para la Protección de los Sujetos Humanos en Investigaciones para Instituciones Internacionales del Departamento de Salud y Servicios Humanos de los Estados Unidos de América, el cual es renovado periódicamente ${ }^{2}$. Entre las funciones del CEIB están:

1. Dictaminar ética y metodológicamente protocolos de investigación de la FCM y otras facultades de la UNAH e instituciones ajenas que tengan relación con la universidad, entre ellas la Secretaría de Salud, Escuela Agrícola Panamericana, OPS/OMS, y universidades de EUA.

2. Capacitar en Buenas Prácticas Clínicas e Integridad Científica tanto en línea, como presencial.

\section{3.} Relaciones de colaboración con instituciones nacionales e internacionales: Biblioteca Médica Nacional, OPS/OMS, Universidad de Miami, Universidad Cayetano de Heredia (Perú), Universidad de Sao Pablo (Brasil), The Global Health Network (Oxford), UNESCO (París).

4. Asesorar y apoyar la gestión de otros comités de menor experiencia o de más reciente creación del país. 
A raíz de la pandemia COVID-19, y debido al confinamiento obligatorio vivido en el país, el CEIB suspendió la revisión en forma física, restableciendo la revisión por correo electrónico en abril de 2020, esto disminuyó la revisión de protocolos en un 50\%, pese a que se contaba con la plataforma ProEthos que es una herramienta para someter y revisar protocolos, conocida desde ya varios años por los diferentes comités de ética en investigación en el país, esta al igual que en otros países de la región no se ha logrado implementar en los comités y entre los miembros de la red ${ }^{7}$. El CEIB dictaminó hasta la fecha más de 25 protocolos en el tema COVID-19, tratando de disminuir los tiempos de revisión, seguir capacitándonos en línea sobre los dilemas éticos que han surgido y el abordaje que recomiendan los comités internacionales de Ética.

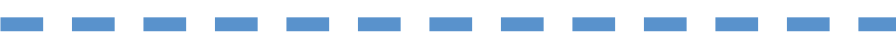

Desde la academia se ha impulsado la creación de comités de ética institucionales, función que debería rectorar la Secretaría de Salud

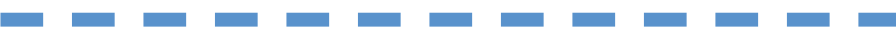

\section{La bioética durante la pandemia COVID 19}

El 30 de enero del 2020 el COVID-19 fue declarado emergencia de Salud Pública ${ }^{3}$, y el 11 de marzo pandemia mundial ${ }^{4}$. Durante estos 19 meses de pandemia, los sistemas de salud del mundo se vieron colapsados, hubo además un franco deterioro en la educación tanto pública como privada, de las economías y de la seguridad de la población, aunado a ello todos los problemas éticos que se han evidenciado desde el diagnostico de una enfermedad nueva, como la selección de pacientes a tratar, los tratamientos experimentales, entre otros. "la ivermectina hasta los productos sanguíneos como el plasma convaleciente, intervenciones que han demostrado ser seguras y eficaces autorizadas para una afección distinta de la COVID-19, la hidroxicloroquina, así como intervenciones cuya eficacia no se ha demostrado, ni han sido autorizadas para otra afección, el remdesivir. Dado que la seguridad y eficacia de estas intervenciones no han sido probadas aún para COVID-19, se desconoce su perfil de riesgo-beneficio ${ }^{5}$. Otro punto importante a considerar es la decisión individual a vacunarse o no, en donde se pone en juego valores morales como el bien común en detrimento del bien individual, el derecho de la libre movilización por el confinamiento obligado, entre otros. Si analizamos todo ello se necesita un marco de referencia para establecer y apoyar decisiones que, aunque legales, carecen de una base ética que se traduzca claramente a los individuos y poblaciones afectados ${ }^{6}$. Según una publicación de OPS sobre orientación y estrategias para agilizar la revisión y supervisión éticas de investigaciones relacionadas con COVID-19 se deben crear mecanismos y procedimientos flexibles y alternativos para la revisión y la supervisión éticas que mejor se adecúen a las características del país ${ }^{8}$.

\section{Red de Comités de Ética de Honduras (REDCEIH)}

En el año 2012, se llevaron a cabo el I y II encuentro de comités de ética de investigación del país, actualmente hay más de 15 comités unos más fortalecidos que otros. En el año 2013, CEIB-FCM-UNAH CEIB somete la propuesta "Fortaleciendo la capacidad investigadora a través del Comité de Ética en Investigación Biomédica (CEIB) de la Facultad de Ciencias Médicas, UNAH. Diciembre 2013-diciembre 2014" a la OMS/OPS / TDR/DQR. Esta iniciativa se concretó en acuerdos de trabajo con otros comités nacionales e instituciones 
internacionales entre ellas la Universidad de Miami (CITI-PROGRAM), y Universidad Cayetano Heredia Perú (Curso Integridad Científica). En el año 2015, el CEIB somete una nueva propuesta "Establecimiento de la Red de Comités de Ética en Investigación (REDCEIH) y fortalecimiento de la investigación ética en Honduras. proyecto que culminó con la creación de la Red de Comités de Ética de la Investigación de Honduras (REDCEIH) desde la cual se podría implementar esta nueva visión de la bioética en el país, conformar una masa crítica de expertos y profesionales versados que apoyen a los tomadores de decisiones y población en general a dar respuesta a los dilemas éticos del mundo actual.

\section{La plataforma ProEthos que es} una herramienta para someter

y revisar protocolos, conocida desde ya varios años por los diferentes comités de ética en investigación del país

Durante el periodo de pandemia COVID-19, el funcionamiento de la red ha sido un canal de información sobre el tema de la ética y la bioética en tiempos de pandemia, creemos que es importante reactivar las actividades de la misma para poder dar una respuesta estandarizada, ya que actualmente cada uno de los comités de la red toma decisiones según sus propios criterios, es así que no sabemos en el país el número de estudios realizados sobre COVID-19 ni qué medidas han implementado durante esta pandemia, el CEIB, es parte de la Red de Comités de Ética de Centroamérica y República Dominicana y participó en el estudio "Fortalecimiento de la gobernanza y regulación ética de la investigación en América Central y la República Dominicana", diciembre de
2020 hasta marzo de 2021; en respuesta a la pandemia de COVID-19. El resultado previsto de este proyecto es una declaración de política sobre la gobernanza y la reglamentación de la ética de la investigación como respuesta al COVID-19, lo que será muy importante para la región.

\section{Comisión Nacional de Bioética-Honduras}

En el año 2018, el CEIB con apoyo de UNESCO llevo a cabo la primera reunión de consulta sobre la creación del Comisión Nacional de Bioética (CNB) en Honduras, con la participación de más de 22 instituciones académicas públicas y privadas, sociedad civil, fundaciones privadas sin fines de lucro, Secretaría de Salud, iglesias, grupos étnicos, entre otras, y dos expertos de UNESCO (Oficina Montevideo). Durante los días 21 y 22 de marzo de 2019 se dieron los insumos y recomendaciones por parte de UNESCO, teniendo como producto final el primer borrador de Propuesta de la creación del Comité Nacional de Bioética, esta propuesta ha sido socializada en diferentes ámbitos tanto en lo académico como grupos de sociedad civil (Universidad Valle de Sula, miembros de la red de comités de Ética de investigación de Honduras, Colegios Profesionales). (https://www.latribuna. hn/2019/11/17/comite-nacional-de-bioetica-dehonduras/). Como consecuencia de la pandemia, la iniciativa se ha quedado en pausa, esperando poder retomarla a futuro.

\section{CONCLUSIONES}

Entre los retos que Honduras enfrenta en el contexto de bioética debe contar con una verdadera regulación sobre la ética y bioética de la investigación, además de las regulaciones de los comités, estandarizar los procedimientos de manejo de revisión, contar además 
con espacios de análisis y reflexión donde se aborden problemas reales como la violencia, la migración, pobreza, enfermedades desatendidas, enfermedades crónicas etc , desde una perspectiva, global, inclusiva, reflexiva y critica, seguir fortaleciendo el trabajo en red nacional REDCEIH e internacional Red CEI CAC e impulsar la creación de la Comisión Nacional de Bioética. Todo ello para apoyar a los tomadores de decisiones y población en general, a dar respuesta a los dilemas bioéticos que vivimos en el mundo actual y de Honduras en particular.

\section{REFERENCIAS BIBLIOGRÁFICAS}

\section{1.}

Honduras. Agencia de Regulación Sanitaria ARSA. Disposiciones para la Regulación y Control Sanitario de los Ensayos Clínicos en Seres Humanos o Muestras Biológicas de éstos en Fase I, II, III y IV y las cuotas de recuperación por servicios prestados. Acuerdo No. 041-2020. Diario Oficial La Gaceta No. 35,419. [Internet]. 27 de octubre de 2020. [citado 27 octubre 2021]. Disponible en: https://arsa.gob.hn/public/ archivos/AcuerdoNo.041-2020-EnsayosClinicos.pdf

\section{2.}

Asociación Médica Mundial. Declaración de HELSINKI de la AMM: Principios éticos para las investigaciones médicas en seres humanos. FerneyVoltaire, Francia: AMM: 2021.

\section{3.}

Consejo de Organizaciones Internacionales de las Ciencias Médicas,Organización Mundial de la Salud. Pautas éticas internacionales para la investigación relacionada con la salud con seres humanos. [Internet]. $4^{\mathrm{a}}$ ed. Ginebra: CIOMS,OMS;2016. [ citado 27 octubre 2021].Disponible en : https://cioms.ch/wp-content/ uploads/2018/01/CIOMS-EthicalGuideline_SP WEB.pdf

\section{Financiamiento y reconocimiento institucional}

El autor es docente de la Unidad de Investigación de la Facultad de Ciencias Médicas (UNAH) y miembro del Comité de Ética en Investigación Biomédica, Facultad de Ciencias Médicas (UNAH).

\section{AGRADECIMIENTOS}

A la Dra. Jackeline Alger, por hacer una lectura critica a este artículo.

\section{4}

Espinoza E, Alger J, Padgett D, Gonzales M . Comité de Ética en Investigación Biomédica (CEIB) de la Facultad de Ciencias Médicas, Universidad Nacional Autónoma de Honduras: Experiencia 2000 - 2010. Rev Med Hondur. 2011;79: 98-102.

\section{5.}

Organización Mundial de la Salud. Declaración del director general de la OMS sobre el Comité de Emergencia del RSI sobre el nuevo coronavirus (2019nCoV). [Internet]. Ginebra: OMS: 2021. [ citado 27 octubre 2021]. Disponible en: https://www.who.int/ $\mathrm{dg} /$ speeches/detail/who-director general-s-statementon-ihr-emergency-committee-on-novel-coronavirus(2019-ncov) [Consultado el 24 de octubre de 2021].

\section{6.}

Organización Mundial de la Salud. Palabras de apertura del director general de la OMS en la rueda de prensa sobre la COVID-19, 2020. [Internet]. Ginebra: OMS: 2021. [ citado 27 octubre 2021]. Disponible en: https://www.who.int/dg/speeches/detail/who-directorgeneral-s-opening-remarks-at-the-media-briefing-oncovid-19-11-march-2020 [Consultado el 24 de octubre de 2021]. 


\section{7.}

Organización Panamericana de la Salud. Uso de emergencia de intervenciones no probadas y fuera del ámbito de la investigación: orientación ética para la pandemia de Covid-19”, 2021. [Internet]. Ginebra: OMS: 2021. [ citado 27 octubre 2021]. Disponible en: https://iris.paho.org/handle/10665.2/52430

\section{8.}

Lin CA, Grunspun H, Nazareth JC, Oliveira RA. Principios y valores bioéticos durante las pandemias. Clínicas (Sao Paulo). 2020;75:e2154. doi: 10.6061/ clinics/2020/e2154.

\section{9.}

Sánchez A, Casado M, Saénz C, Ying A. Bioética en la era del covid-19. Apanac [Internet]. 2021 [citado 25 octubre 2021]; 134-42. Disponible en: https://revistas. utp.ac.pa/index.php/apanac/article/view/3178

\section{0.}

Organización Panamericana de la Salud. Orientación y estrategias para agilizar la revisión y supervisión éticas de investigaciones relacionadas con COVID-19. 2020. [Internet]. Washington D.C.: OPS; 2021. [ citado 27 octubre 2021]. Disponible en: https://iris.paho.org/ handle/10665.2/52090 